\title{
EXTENSIONS OF CONTINUOUS FUNCTIONS FROM DENSE SUBSPACES
}

\author{
ROBERT L. BLAIR
}

\begin{abstract}
Let $X$ and $Y$ be topological spaces, let $S$ be a dense subspace of $X$, and let $f: S \rightarrow Y$ be continuous. When $Y$ is the real line $\mathbf{R}$, the Lebesgue sets of $f$ are used to provide necessary and sufficient conditions in order that the (bounded) function $f$ have a continuous extension over $X$. These conditions yield the theorem of Taimanov (resp. of Engelking and of Blefko and Mrówka) which characterizes extendibility of $f$ for $Y$ compact (resp. realcompact). In addition, an extension theorem of Blefko and Mrówka is sharpened for the case in which $X$ is first countable and $Y$ is a closed subspace of $\mathbf{R}$.
\end{abstract}

We first quote (in Theorem 1) two basic results concerning extension of a continuous function from a dense subspace of a topological space. Theorem $1 \mathrm{~A}$ is due to Taĭmanov [10] (see also [5, Theorem 3.2.1]) and, in dual form, to Eilenberg and Steenrod [3, Lemma 10.9.6] (cf. [5, Exercise 3.2A]). Theorem 1B is due, independently, to Engelking [4, Theorem 2] and to Blefko and Mrówka [2, Theorem A]. (Theorem A of [2] includes the unneeded hypothesis that $X$ is $T_{1}$.) For additional results on extension of continuous functions from dense subspaces, see McDowell [7].

TheOrem 1. Let $X$ and $Y$ be topological spaces, let $S$ be a dense subspace of $X$, and let $f: S \rightarrow Y$ be continuous.

A (Taimanov). If $Y$ is compact (Hausdorff), then these are equivalent:

(1) $f$ extends continuously over $X$.

(2) If $F_{1}$ and $F_{2}$ are disjoint closed subsets (or, alternatively, zero-sets) of $Y$, then $f^{-1}\left(F_{1}\right)$ and $f^{-1}\left(F_{2}\right)$ have disjoint closures in $X$.

B (ENGELKING AND BlefKo-MróWKA). If $Y$ is realcompact, then these are equivalent:

(1) $f$ extends continuously over $X$.

(2) If $\left\{F_{n}\right\}_{n=1}^{\infty}$ is any sequence of closed subsets (or, alternatively, zero-sets) of $Y$ with $\cap_{n=1}^{\infty} F_{n}=\varnothing$, then $\cap_{n=1}^{\infty} \mathrm{cl}_{X} f^{-1}\left(F_{n}\right)=\varnothing$.

By a zero-set is meant the set of zeros of a real-valued continuous function. For the theory of realcompact spaces, see Gillman and Jerison [6].

Received by the editors July $31,1974$.

AMS (MOS) subject classifications (1970). Primary 54C05, 54C20, 54C30; Secondary 54C45, 54D30, 54D60.

Key words and phrases. Continuous function, real-valued continuous function, continuous extension, dense subspace, compact space, realcompact space, zero-set, Lebesgue set, $C^{*}$ embedded, $C$-embedded, first countable space. 
In this note we obtain a sharper version of Theorem 1A (resp. 1B) for the special case in which $f$ is a bounded continuous function (resp. continuous function) from $S$ into the real line $\mathbf{R}$; this is Theorem 2 below. Theorem 1, in turn, will follow readily from Theorem 2 . We also include a sharpening (for real-valued functions) of a theorem of Blefko and Mrówka concerning extension of a continuous function from a dense subspace of a first countable space [2, Theorem D] (see Theorem 3 below).

If $X$ is a topological space, then $C(X)$ (resp. $\left.C^{*}(X)\right)$ will denote the set of all continuous (resp. bounded continuous) real-valued functions on $X$. If $f \in C(X)$ and $a \in \mathbf{R}$, we set

$$
L_{a}(f)=\{x \in X: f(x) \leqslant a\}, \quad L^{a}(f)=\{x \in X: f(x) \geqslant a\} .
$$

Sets of the form $L_{a}(f)$ or $L^{a}(f)$ are Lebesgue sets of $f$. The point of Theorem 2 (which may be viewed as an analogue of $[6,1.18]$ ) is that it characterizes extendibility of $f$ in terms of the Lebesgue sets of $f$. (Theorem 2 is thus a fragment of a general program whereby real-valued functions are studied by means of their Lebesgue sets; see, e.g., [8], [9], and [1, §\$2-3]. Other aspects of this program will be treated by the author elsewhere.)

THEOREM 2. Let $S$ be a dense subspace of a topological space $X$, let $f \in C(S)$, and consider these conditions on $f$ :

(a) $f$ extends continuously over $X$.

(b) Disjoint Lebesgue sets of $f$ have disjoint closures in $X$.

(c) $\cap_{n=1}^{\infty} \mathrm{cl}_{X}\left(L_{-n}(f) \cup L^{n}(f)\right)=\varnothing$.

Then (a) is equivalent to the conjunction of (b) and (c); and if $f \in C^{*}(S)$, (a) is equivalent to (b).

Proof. First assume (a), so that $f=g \mid S$ for some $g \in C(X)$. To verify (b), we need only note that if $a<b$, then

$$
\mathrm{cl}_{X} L_{a}(f) \cap \mathrm{cl}_{X} L^{b}(f) \subset L_{a}(g) \cap L^{b}(g)=\varnothing .
$$

To verify (c), let $p \in X$, choose $n \geqslant|g(p)|+1$, and note that $\{x \in X$ : $|g(x)-g(p)|<1\}$ is a neighborhood of $p$ in $X$ which misses $L_{-n}(f)$ $\cup L^{n}(f)$.

Observe next that, to verify (a), it suffices to show that $f$ has an extension $f_{p} \in C(S \cup\{p\}$ ) for every $p \in X$. (For then $g: X \rightarrow \mathbf{R}$ can be defined by $g=f$ on $S$ and $g(p)=f_{p}(p)$ for $p \in X-S$; and since $S$ is dense in $X, g$ is continuous $[6,6 \mathrm{H}]$.) For the remainder of the proof, we may therefore assume that $X=S \cup\{p\}$, with $p \notin S$.

Assume (b) and (c), and let $A=\left\{s \in \mathbf{R}: p \in \operatorname{cl}^{s}(f)\right\}, B=\{r \in \mathbf{R}: p$ $\left.\in \operatorname{cl} L_{r}(f)\right\}$. Since $X=\mathrm{cl} S$, (c) implies that there is an $n$ such that $p \in \operatorname{cl} L_{n}(f) \cap \mathrm{cl} L^{-n}(f)$. Hence both $A$ and $B$ are nonempty. Moreover, by (b), we have $s \leqslant r$ for every $s \in A$ and every $r \in B$. Let $s^{*}=\sup A$, $r^{*}=\inf B$, and note that $s^{*} \leqslant r^{*}$. If $s^{*}<r^{*}$, there is $t \in \mathbf{R}$ with $s^{*}<t$ $<r^{*}$; but then $p \notin \operatorname{cl}\left(L_{t}(f) \cup L^{t}(f)\right)=\operatorname{cl} S$, a contradiction. Thus $s^{*}$ $=r^{*}$. Define $g: X \rightarrow \mathbf{R}$ by $g=f$ on $S$ and $g(p)=s^{*}=r^{*}$. We verify that $g$ is continuous at each point of $X$ : 
Let $x \in X, \epsilon>0$, and $V=(g(x)-\epsilon, g(x)+\epsilon)$.

Case 1. $x=p$. Let $U=X-\operatorname{cl}\left(L_{g(p)-\epsilon}(f) \cup L^{g(p)+\epsilon}(f)\right)$. Since $g(p)-\epsilon$ $<s \leqslant r<g(p)+\epsilon$ for some $s \in A$ and $r \in B$, it follows from (b) that $p \in U$, and clearly $g(U) \subset V$.

Case 2. $x \in S$. There is an open neighborhood $W$ of $x$ in $S$ with $f(W) \subset(g(x)-(\epsilon / 3), g(x)+(\epsilon / 3))$. Write $W=S \cap G$, with $G$ open in $X$. If $p \notin G$, then $g(G)=f(W) \subset V$, so we may assume that $p \in G$. Now $W \subset L^{g(x)-(\epsilon / 3)}(f)$, so we have $p \in \operatorname{cl} G=\operatorname{cl} W \subset \operatorname{cl}^{g(x)-(\epsilon / 3)}(f)$. If $g(p)$ $<g(x)-(2 \epsilon / 3)$, there is $r \in B$ with $r<g(x)-(2 \epsilon / 3)$. But then $p$ $\in \operatorname{cl} L_{r}(f) \subset \operatorname{cl} L_{g(x)-(2 \epsilon / 3)}(f)$, which is contrary to (b). Thus $g(p) \geqslant g(x)$ $-(2 \epsilon / 3)$, and, similarly, $g(p) \leqslant g(x)+(2 \epsilon / 3)$. We conclude that $g(G) \subset V$, and hence $g$ is a continuous extension of $f$.

To complete the proof, note that if $f \in C^{*}(S)$ and if $n>|f|$, then $L_{-n}(f) \cup L^{n}(f)=\varnothing$, so (c) holds automatically.

Proof of Theorem 1. A(1) $\Rightarrow \mathrm{A}(2)$. Assume that $f=g \mid S$, with $g: X \rightarrow Y$ continuous. If $F_{1}$ and $F_{2}$ are disjoint closed subsets of $Y$, then

$$
\operatorname{cl}_{X} f^{-1}\left(F_{1}\right) \cap \operatorname{cl}_{X} f^{-1}\left(F_{2}\right) \subset g^{-1}\left(F_{1}\right) \cap g^{-1}\left(F_{2}\right)=\varnothing .
$$

Similarly, $\mathrm{B}(1) \Rightarrow \mathrm{B}(2)$.

$\mathrm{A}(2) \Rightarrow \mathrm{A}(1)$ (resp. $\mathrm{B}(2) \Rightarrow \mathrm{B}(1))$. We may assume that the compact (resp. realcompact) space $Y$ is a closed subspace of a product $Y^{\prime}=\prod_{\alpha \in I} Y_{\alpha}$, where $Y_{\alpha}=[0,1]$ (resp. $\left.Y_{\alpha}=\mathbf{R}\right)$ for each $\alpha \in I($ see $[6,11.12])$. Let $f_{\alpha}=\left(\operatorname{pr}_{\alpha} \mid Y\right) \circ f$, where $\operatorname{pr}_{\alpha}$ is the projection of the product $Y^{\prime}$ of index $\alpha$. It suffices to show that each $f_{\alpha}$ satisfies (b) (resp. (b) and (c)) of Theorem 2. (For then $f_{\alpha}$ has a continuous extension $g_{\alpha}: X \rightarrow Y_{\alpha}$, the diagonal map $g=\Delta_{\alpha \in I} g_{\alpha}: X \rightarrow Y^{\prime}$ is continuous, $g=f$ on $S$, and $g(X)=g(\operatorname{cl} S) \subset \operatorname{cl} g(S) \subset Y$; cf. [4, Lemma 1].) For each $a \in \mathbf{R}$, let $Z_{a}=Y \cap \operatorname{pr}_{\alpha}^{-1}((-\infty, a]), Z^{a}=Y \cap \operatorname{pr}_{\alpha}^{-1}([a,+\infty))$. Note that $Z_{a}$ and $Z^{a}$ are zero-sets in $Y$ and that $L_{a}\left(f_{\alpha}\right)=f^{-1}\left(Z_{a}\right), L^{a}\left(f_{\alpha}\right)$ $=f^{-1}\left(Z^{a}\right)$. It follows from (the zero-set formulation of) either $\mathrm{A}(2)$ or $\mathrm{B}(2)$ that if $a<b$, then $L_{a}\left(f_{\alpha}\right)$ and $L^{b}\left(f_{\alpha}\right)$ have disjoint closures in $X$; hence (b) holds in either case. Moreover, $\bigcap_{n=1}^{\infty}\left(Z_{-n} \cup Z^{n}\right)=\varnothing$, so (the zero-set formulation of) $\mathrm{B}(2)$ implies that $\cap_{n=1}^{\infty} \mathrm{cl}_{X}\left(L_{-n}\left(f_{\alpha}\right) \cup L^{n}\left(f_{\alpha}\right)\right)=\varnothing$. Thus (c) holds, and the proof is complete.

We note that, by a similar argument, Theorem $\mathrm{C}$ of [2] is also an easy consequence of Theorem 2.

A subset $S$ of a topological space $X$ is $C^{*}$-embedded (resp. $C$-embedded) in $X$ in case every $f \in C^{*}(S)$ (resp. $f \in C(S)$ ) has a continuous extension over $X$. The following corollary (formulated and proved in [6, Theorems 6.4 and 8.6] in the context of Tychonoff spaces; cf. [11]) is an immediate consequence of either Theorem 1 or Theorem 2 .

COROLlary. Let $S$ be a dense subspace of a topological space $X$.

A. These are equivalent:

(1) $S$ is $C^{*}$-embedded in $X$.

(2) Any two disjoint zero-sets in $S$ have disjoint closures in $X$.

B. These are equivalent:

(1) $S$ is $C$-embedded in $X$. 
(2) If a countable family of zero-sets in $S$ has empty intersection, then their closures in $X$ have empty intersection.

It is known that (the closed set formulation of) Theorem 1A holds if $Y$ is merely Tychonoff, provided that $X$ is first countable [2, Theorem D]. For the special case in which $Y$ is a closed subset of $\mathbf{R}$, we can apply Theorem 2 to sharpen this result as follows:

THEOREM 3. Let $S$ be a dense subspace of a topological space $X$, assume each $p \in X-S$ has a countable base of neighborhoods, let $Y$ be a closed subspace of $\mathbf{R}$, and let $f: S \rightarrow Y$ be continuous. Then these are equivalent:

(1) $f$ extends continuously over $X$.

(2) If $F_{1}$ and $F_{2}$ are disjoint countable closed subsets of $Y$, then $f^{-1}\left(F_{1}\right)$ and $f^{-1}\left(F_{2}\right)$ have disjoint closures in $X$.

Proof. (1) $\Rightarrow(2)$. This follows as in the proof of $A(1) \Rightarrow A(2)$ of Theorem 1.

(2) $\Rightarrow(1)$. It suffices to show that $f$ (regarded as a function from $S$ into $\mathbf{R}$ ) has a continuous extension $g: X \rightarrow \mathbf{R}$. (For then $g(X)=g(\operatorname{cl} S) \subset \operatorname{cl} g(S)$ $\subset Y$.) We verify that $f: S \rightarrow \mathbf{R}$ satisfies (b) and (c) of Theorem 2 .

Suppose first that (b) fails. Then for some $a<b$ there exists $p \in \operatorname{cl} L_{a}(f)$ $\cap \mathrm{cl} L^{b}(f)$. Obviously $p \in X-S$, so $p$ has a countable base of neighborhoods $\left\{U_{n}\right\}_{n=1}^{\infty}$. Choose $c \in \mathbf{R}$ with $a<c<b$. We shall show that there is a countable closed subset $F_{1}$ of $\mathbf{R}$ with $p \in \mathrm{cl} f^{-1}\left(F_{1} \cap Y\right)$ and $F_{1} \subset(c,+\infty)$. Let $s^{*}=\sup \left\{s \in \mathbf{R}: p \in \operatorname{cl} L^{s}(f)\right\}$.

Case 1. $s^{*}<+\infty$. For each $n>0$, we have $c \vee\left(s^{*}-(1 / n)\right)<s^{*}$, so there is $s(n) \in \mathbf{R}$ with $p \in \mathrm{cl} L^{s(n)}(f)$ and $c \vee\left(s^{*}-(1 / n)\right)<s(n)$. Moreover, $p \notin \mathrm{cl} L^{s^{*}+(1 / n)}(f)$, so there exists a point $x_{n}$ with

$$
x_{n} \in U_{n} \cap\left(X-\operatorname{cl} L^{s^{*}+(1 / n)}(f)\right) \cap L^{s(n)}(f) .
$$

Let $F_{1}=\left\{f\left(x_{n}\right): n=1,2, \ldots\right\} \cup\left\{s^{*}\right\}$. Since $\left|f\left(x_{n}\right)-s^{*}\right|<1 / n$, we have $f\left(x_{n}\right) \rightarrow s^{*}$, and hence $F_{1}$ is closed in R. Clearly $p \in \operatorname{cl} f^{-1}\left(F_{1} \cap Y\right)$ and $F_{1} \subset(c,+\infty)$.

Case 2. $s^{*}=+\infty$. Construct a sequence $\left\{x_{n}\right\}_{n=1}^{\infty}$ as follows: Pick $x_{1}$ $\in U_{1} \cap L^{b}(f)$; and if $x_{1}, \ldots, x_{n-1}$ have already been chosen with $x_{i}$ $\in U_{i} \cap L^{b}(f)$ and $f\left(x_{i}\right)>f\left(x_{i-1}\right) \vee i(i=2, \ldots, n-1)$, choose $s \in \mathbf{R}$ with $p \in \operatorname{cl} L^{s}(f)$ and $f\left(x_{n-1}\right) \vee n<s$, and pick $x_{n} \in U_{n} \cap L^{s}(f)$. Then $\left\{f\left(x_{n}\right)\right\}_{n=1}^{\infty}$ is strictly increasing and $f\left(x_{n}\right) \rightarrow+\infty$, so $F_{1}=\left\{f\left(x_{n}\right): n=1\right.$, $2, \ldots\}$ is closed in $\mathbf{R}$. Moreover, $x_{n} \in U_{n} \cap L^{b}(f)$ for all $n$, so we have $p \in \mathrm{cl} f^{-1}\left(F_{1} \cap Y\right)$ and $F_{1} \subset(c,+\infty)$.

Similarly, there is a countable closed subset $F_{2}$ of $\mathbf{R}$ with

$$
p \in \operatorname{cl} f^{-1}\left(F_{2} \cap Y\right) \text { and } F_{2} \subset(-\infty, c) \text {. }
$$

Thus (2) fails.

Suppose next that (c) of Theorem 2 fails. Then there exists $p$ $\in \cap_{n=1}^{\infty} \mathrm{cl}_{X}\left(L_{-n}(f) \cup L^{n}(f)\right)$, and clearly $p \in X-S$. Let $\left\{U_{n}\right\}_{n=1}^{\infty}$ be a countable base of neighborhoods at $p$ with $U_{n} \supset U_{n+1}$ for each $n$. Pick 
$x_{1} \in U_{1} \cap\left(L_{-1}(f) \cup L^{1}(f)\right)$; and if $x_{1}, \ldots, x_{n-1}$ have already been chosen with $x_{i} \in U_{i} \cap\left(L_{-i}(f) \cup L^{i}(f)\right)$ and $\left|f\left(x_{i}\right)\right|>\left|f\left(x_{i-1}\right)\right| \vee i(i=2, \ldots, n$ - 1), let $m(n)$ be the least integer $>\left|f\left(x_{n-1}\right)\right| \vee n$, and pick $x_{n} \in U_{n}$ $\cap\left(L_{-m(n)}(f) \cup L^{m(n)}(f)\right)$. We thus construct a sequence $\left\{x_{n}\right\}_{n=1}^{\infty}$ with $x_{n}$ $\in U_{n},\left\{\left|f\left(x_{n}\right)\right|\right\}_{n=1}^{\infty}$ strictly increasing, and $\left|f\left(x_{n}\right)\right| \rightarrow \infty$. Let

$$
\begin{aligned}
& F_{1}=\left\{r \in \mathbf{R}:|r|=\left|f\left(x_{n}\right)\right| \text { for some } n, n \text { odd }\right\}, \\
& F_{2}=\left\{r \in \mathbf{R}:|r|=\left|f\left(x_{n}\right)\right| \text { for some } n, n \text { even }\right\} .
\end{aligned}
$$

Then $F_{1}$ and $F_{2}$ are disjoint countable closed subsets of $\mathbf{R}$ with

$$
p \in \operatorname{cl} f^{-1}\left(F_{1} \cap Y\right) \cap \operatorname{cl} f^{-1}\left(F_{2} \cap Y\right),
$$

so (2) fails once again. The proof is therefore complete.

We leave open the question of possible generalizations of Theorem 3 (for Tychonoff spaces $Y$ that are not necessarily closed subspaces of $\mathbf{R}$ ).

\section{REFERENCES}

1. R. L. Blair, Filter characterizations of $z-, C^{*}$-, and $C$-embeddings, Fund. Math. (to appear).

2. R. Blefko and S. Mrówka, On the extensions of continuous functions from dense subspaces, Proc. Amer. Math. Soc. 17 (1966), 1396-1400. MR 34 \# 1989.

3. S. Eilenberg and N. Steenrod, Foundations of algebraic topology, Princeton Univ. Press, Princeton, N. J., 1952. MR 14, 398.

4. R. Engelking, Remarks on real-compact spaces, Fund. Math. 55 (1964), 303-308. MR 31 \#4000.

5. - Outline of general topology, PWN, Warsaw, 1965; English transl., North-Holland, Amsterdam; Interscience, New York, 1968. MR 36 \#4508; MR 37 \#836.

6. L. Gillman and M. Jerison, Rings of continuous functions, University Series in Higher Math., Van Nostrand, Princeton, N. J., 1960. MR 22 \#6994.

7. R. H. McDowell, Extension of functions from dense subspaces, Duke Math. J. 25 (1958), 297-304. MR 20 \#4251.

8. S. Mrówka, On some approximation theorems, Nieuw Arch. Wisk. 16 (1968), 94-111. MR 39 \#6251.

9. - Characterization of classes of functions by Lebesgue sets, Czechoslovak Math. J. 19 (94) (1969), 738-744. MR 40 \#1543.

10. A. D. Taĭmanov, On the extension of continuous mappings of topological spaces, Mat. Sb. 31 (73) (1952), 459-462. (Russian) MR 14, 395.

11. B. Z. Vulih, On the extension of continuous functions in topological spaces, Mat. Sb. 30 (72) (1952), 167-170. (Russian) MR 14, 70.

Department of Mathematics, Ohio University, Athens, Ohio 45701 